\title{
Positive Solutions for Singular $p$-Laplacian Fractional Differential System with Integral Boundary Conditions
}

\author{
Liping Wang, ${ }^{1}$ Zongfu Zhou, ${ }^{1}$ and Hui Zhou ${ }^{2}$ \\ ${ }^{1}$ School of Mathematical Science, Anhui University, Hefei, Anhui 230601, China \\ ${ }^{2}$ Department of Mathematics and Statistics, Hefei Normal University, Hefei, Anhui 230601, China
}

Correspondence should be addressed to Zongfu Zhou; zhouzf12@126.com and Hui Zhou; zhouhui0309@126.com

Received 17 January 2014; Accepted 1 April 2014; Published 27 April 2014

Academic Editor: Ivanka Stamova

Copyright (C) 2014 Liping Wang et al. This is an open access article distributed under the Creative Commons Attribution License, which permits unrestricted use, distribution, and reproduction in any medium, provided the original work is properly cited.

\begin{abstract}
This paper investigates the existence of positive solutions for a class of singular $p$-Laplacian fractional differential equations with integral boundary conditions. By using the Leggett-Williams fixed point theorem, the existence of at least three positive solutions to the boundary value system is guaranteed.
\end{abstract}

\section{Introduction}

This paper investigates the singular $p$-Laplacian fractional boundary value problem:

$$
\begin{gathered}
D_{0^{+}}^{\beta}\left(\phi_{p_{1}}\left(D_{0^{+}}^{\alpha} u(t)\right)\right) \\
=f_{1}\left(t, u(t), v(t), D_{0^{+}}^{\mu} u(t), D_{0^{+}}^{v} v(t)\right), \quad 0<t<1, \\
D_{0^{+}}^{\beta}\left(\phi_{p_{2}}\left(D_{0^{+}}^{\alpha} v(t)\right)\right) \\
=f_{2}\left(t, u(t), v(t), D_{0^{+}}^{\mu} u(t), D_{0^{+}}^{v} v(t)\right), \quad 0<t<1, \\
u(0)=u^{\prime}(0)=u^{\prime \prime}(0)=0, \\
u^{\prime \prime}(1)=(\alpha-1)(\alpha-2) \int_{0}^{1} u(s) d \xi_{1}(s), \\
D_{0^{+}}^{\alpha} u(0)=0, \quad \phi_{p_{1}}\left(D_{0^{+}}^{\alpha} u(1)\right)=\int_{0}^{1} \phi_{p_{1}}\left(D_{0^{+}}^{\alpha} u(s)\right) d \eta_{1}(s), \\
v(0)=v^{\prime}(0)=v^{\prime \prime}(0)=0, \\
v^{\prime \prime}(1)=(\alpha-1)(\alpha-2) \int_{0}^{1} v(s) d \xi_{2}(s), \\
D_{0^{+}}^{\alpha} v(0)=0, \quad \phi_{p_{2}}\left(D_{0^{+}}^{\alpha} v(1)\right)=\int_{0}^{1} \phi_{p_{2}}\left(D_{0^{+}}^{\alpha} v(s)\right) d \eta_{2}(s),
\end{gathered}
$$

where $1<\beta \leq 2,3<\alpha \leq 4$ and $5<\alpha+\beta \leq 6$, $0<\mu \leq 1,1<\nu<2$ are real numbers. $\phi_{p_{i}}(x)=|x|^{p_{i}-2} x$, $p_{i}>1, \phi_{q_{i}}=\phi_{p_{i}}^{-1}, 1 / p_{i}+1 / q_{i}=1$, and $\xi_{i}, \eta_{i}:[0,1] \rightarrow R^{+}$ are nondecreasing functions of bounded variation $i=1,2$. $D^{\alpha}$ and $D^{\beta}$ are the standard Riemann-Liouville fractional derivatives of order $\alpha, \beta$, respectively, and the integrals in (1) are Riemann-Stieltjes integrals. Here $f_{i}:[0,1] \times \Omega \rightarrow$ $\mathbb{R}^{+}$is Carathéodory function; that is, $f_{i}$ satisfies the local Carathéodory condition on $[0,1] \times \Omega$, and $f_{i}\left(t, x_{1}, x_{2}, x_{3}, x_{4}\right)$ may be singular at the value 0 of all its space variables $x_{1}, x_{2}, x_{3}, x_{4}, i=1,2$, in which $\Omega=(0, \infty)^{4}, \mathbb{R}^{+}=[0, \infty)$.

We say that $f$ satisfies the local Carathéodory condition on $[0,1] \times \Omega,(f \in \operatorname{Car}([0,1] \times \Omega))$, if

(i) $f\left(\cdot ; x_{1}, x_{2}, x_{3}, x_{4}\right):[0,1] \rightarrow \mathbb{R}^{+}$is measurable for all $\left(x_{1}, x_{2}, x_{3}, x_{4}\right) \in \Omega$

(ii) $f(t ; \cdot, \cdot, \cdot, \cdot): \Omega \rightarrow \mathbb{R}^{+}$is continuous for a.e. $t \in[0,1]$;

(iii) for each compact set $\mathscr{K} \subset \Omega$ there is a function $\varphi_{\mathscr{K}} \in$ $L^{1}[0,1]$ such that $\left|f\left(t, x_{1}, x_{2}, x_{3}, x_{4}\right)\right| \leq \varphi_{\mathscr{K}}(t)$ for a.e. $t \in[0,1]$ and all $\left(x_{1}, x_{2}, x_{3}, x_{4}\right) \in \mathscr{K}$.

A vector $(u, v) \in\left(C^{2}[0,1]\right) \times\left(C^{2}[0,1]\right)$ is called positive solution of system (1) if and only if $(u, v)$ satisfies (1) and $u(t)>0, v(t) \geq 0$ or $v(t)>0, u(t) \geq 0$ for any $t \in[0,1]$.

Fractional differential equation can describe many phenomena in various fields of science and engineering, such 
as control, porous media, electrochemistry, and electromagnetic. For details, see [1-8] and the references therein. There are also a large number of papers dealing with the solvability of nonlinear fractional differential equations. Papers [9-13] discuss fractional boundary value problems with nonlinearities having singularities in space variables.

Paper [14] is concerned with the existence of positive solutions to the following BVP for nonlinear fractional differential equations with boundary conditions involving Riemann-Stieltjes integrals:

$$
\begin{gathered}
D_{0_{+}}^{\alpha} u(t)+f\left(t, u(t), u^{\prime}(t), \ldots, u^{(n-2)}(t)\right)=0, \\
0<t<1, \\
u(0)=u^{\prime}(0)=\cdots=u^{n-2}(0)=0, \\
u^{n-2}(1)=\int_{0}^{1} u^{n-2}(s) d A(s),
\end{gathered}
$$

where $n-1<\alpha \leq n$ and $n \geq 2$ is positive integer, the integrals $\int_{0}^{1} u^{n-2}(s) d A(s)$ are Riemann-Stieltjes integrals, and $f$ is a Carathéodory function on $[0,1] \times \mathbb{R}^{n-1} \rightarrow \mathbb{R}^{+}$. Some existence and multiplicity results of positive solutions are obtained by using the Krasnosel'skii fixed point theorem, the Leray-Schauder nonlinear alternative, and the LeggettWilliams fixed point theorem.

In the past few decades, in order to meet the demands of research, the $p$-Laplacian equation is introduced in some BVP, such as $[15,16]$.

Paper [15] investigates the existence of solutions for the BVP of fractional $p$-Laplacian equation with the following form:

$$
\begin{gathered}
D_{0^{+}}^{\beta}\left(\phi_{p}\left(D_{0^{+}}^{\alpha} x(t)\right)\right)=f\left(t, x(t), D_{0^{+}}^{\alpha} x(t)\right), \\
t \in[0,1], \\
D_{0^{+}}^{\alpha} x(0)=D_{0^{+}}^{\alpha} x(1)=0,
\end{gathered}
$$

where $0<\alpha, \beta \leq 1$, and $1<\alpha+\beta \leq 2$, and $p>1, \phi_{p}(s)=$ $|s|^{p-2} s$ is a $p$-Laplacian operator. $D^{\alpha}$ is a Caputo fractional derivative. A new result on the existence of solutions for the above fractional boundary value problem is obtained, which generalize and enrich some known results to some extent from the literature, by using the coincidence degree theory.

Paper [16] studies the existence of positive solutions of the following singular fourth-order coupled system with integral boundary conditions:

$$
\begin{array}{r}
\left(\phi_{p_{1}}\left(u^{\prime \prime}(t)\right)\right)^{\prime \prime}=\lambda^{p_{1}} a_{1}(t) f_{1}(t, u(t), v(t)), \\
0<t<1, \\
\left(\phi_{p_{2}}\left(v^{\prime \prime}(t)\right)\right)^{\prime \prime}=\mu^{p_{2}} a_{2}(t) f_{2}(t, u(t), v(t)), \\
0<t<1,
\end{array}
$$

$$
\begin{gathered}
u(0)=u(1)=\int_{0}^{1} u(s) d \xi_{1}(s), \\
\phi_{p_{1}}\left(u^{\prime \prime}(0)\right)=\phi_{p_{1}}\left(u^{\prime \prime}(1)\right)=\int_{0}^{1} \phi_{p_{1}}\left(u^{\prime \prime}(s)\right) d \eta_{1}(s), \\
v(0)=v(1)=\int_{0}^{1} v(s) d \xi_{2}(s), \\
\phi_{p_{2}}\left(v^{\prime \prime}(0)\right)=\phi_{p_{2}}\left(v^{\prime \prime}(1)\right)=\int_{0}^{1} \phi_{p_{2}}\left(v^{\prime \prime}(t)\right) d \eta_{2}(s),
\end{gathered}
$$

where $\lambda$ and $\mu$ are positive parameters, $\phi_{p_{i}}(x)=|x|^{p_{i}-2} x, p_{i}>$ $1, \phi_{q_{i}}=\phi_{p_{i}}^{-1}, 1 / p_{i}+1 / q_{i}=1$, and $\xi_{i}, \eta_{i}:[0,1] \rightarrow R^{+}$are nondecreasing functions of bounded variation, $i=1,2$, and the integrals in (4) are Riemann-Stieltjes integrals, $f_{1}:[0,1] \times$ $\mathbb{R}_{0}^{+} \times \mathbb{R}^{+} \rightarrow \mathbb{R}^{+}$and $f_{2}:[0,1] \times \mathbb{R}^{+} \times \mathbb{R}_{0}^{+} \rightarrow \mathbb{R}^{+}$are two continuous functions, and $f_{1}(t, x, y)$ may be singular at $x=0$, while $f_{2}(t, x, y)$ may be singular at $y=0 ; a_{1}, a_{2}:(0,1) \rightarrow \mathbb{R}^{+}$ are continuous and may be singular at $t=0$ and/or $t=1$, in which $\mathbb{R}^{+}=[0, \infty)$ and $\mathbb{R}_{0}^{+}=(0, \infty)$. By using the fixed point theory in cones, explicit range for $\lambda$ and $\mu$ is derived such that for any $\lambda$ and $\mu$ which lie in their respective interval, the existence of at least one positive solution to the boundary value system is guaranteed.

Inspired by above works, our work presented in this paper has the following new features. Firstly, our study is on singular nonlinear differential systems; that is, $f_{i}\left(t, x_{1}, x_{2}, x_{3}, x_{4}\right)$ may be singular at the value 0 of all its space variables $x_{1}, x_{2}, x_{3}, x_{4}$, $i=1,2$, which bring about many difficulties. Secondly, the techniques used in this paper are approximation methods, and a special cone has been developed to overcome the difficulties due to the singularity and to apply the fixed-point theorem. Finally, we discuss the BVP with integral boundary conditions, that is, system (1) including multipoint and nonlocal boundary value problems as special cases. To our knowledge, very few authors studied the existence of positive solutions for $p$-Laplacian fractional differential equation with boundary conditions involving Riemann-Stieltjes integrals. Hence we improve and generalize the results of previous papers to some degree, and so it is interesting and important to study the existence of positive solutions for system (1).

Throughout the paper, $\|u\|_{1}=\int_{0}^{1}|u(t)| d t$ is the norm in the Banach $L^{1}[0,1]$ and $\|u\|_{\infty}=\max _{t \in[0,1]}|u(t)|$ is the norm in the Banach $C[0,1]$. Let $E=C^{2}[0,1]$; then $E$ is a Banach space endowed with the norm $\|u\|_{*}=\max \left\{\|u\|_{\infty},\left\|u^{\prime}\right\|_{\infty},\left\|u^{\prime \prime}\right\|_{\infty}\right\}$. Thus, $(E \times E,\|\cdot\|)$ is a Banach with the norm defined by $\|(u, v)\|=\max \left\{\|u\|_{*},\|v\|_{*}\right\}$ for $(u, v) \in E \times E$.

This paper is organized as follows. In Section 2, we present some results of fractional calculus theory and auxiliary technical lemmas, which are used in the next two sections. Section 3 deals with the approximate problem of (1). We induce the solvability of this problem to the existence of a fixed point of an operator $T_{n}$. By the Leggett-Williams fixed point theorem, the existence of at least three fixed points of $T_{n}$ is obtained. In Section 4, we prove the existence and 
multiplicity of positive solutions of problem (1) by applying the results of Sections 2 and 3.

\section{Preliminaries}

For the convenience of the reader, we present here the necessary definitions and lemmas from fractional calculus theory. These definitions and lemmas can be found in the recent literatures [14, 17-19].

For convenience, we list the following signs and assumptions for system (1).

We note that

(1) $a_{i}=\int_{0}^{1} d \xi_{i}(s), b_{i}=\int_{0}^{1} d \eta_{i}(s), A_{i}=\int_{0}^{1} s^{\alpha-1} d \xi_{i}(s)$, $B_{i}=\int_{0}^{1} s^{\alpha-1} d \eta_{i}(s), i=1,2$;

(2) $e_{\alpha}(s)=s(1-s)^{\alpha-1}, e_{\beta}(s)=s(1-s)^{\beta-1}$.

We assume that

$\left(\mathrm{H}_{1}\right) 0 \leq a_{i}<1,0 \leq b_{i}<1$, and $i=1,2$.

Obviously, $A_{i} \leq a_{i}, B_{i} \leq b_{i}, i=1,2$.

Definition 1 (see [17]). The fractional integral of order $\alpha>0$ of a function $f:(0, \infty) \rightarrow \mathbb{R}$ is defined by

$$
I_{0^{+}}^{\alpha} f(t)=\frac{1}{\Gamma(\alpha)} \int_{0}^{t}(t-s)^{\alpha-1} f(s) d s
$$

provided that the right-hand side exists.

Definition 2 (see [17]). The Riemann-Liouville fractional order derivative of order $\alpha \in(n-1, n]$ of a function $f$ : $(0, \infty) \rightarrow \mathbb{R}$ is defined by

$$
D_{0^{+}}^{\alpha} f(t)=\frac{1}{\Gamma(n-\alpha)} \frac{d^{n}}{d t^{n}} \int_{0}^{t}(t-s)^{n-\alpha-1} f(s) d s
$$

provided that the right-hand side exists.

Lemma 3 (see [17]). Let $\alpha \in(n-1, n]$ and $u \in C(0,1) \cap$ $L^{1}(0,1)$. Then

$$
I_{0^{+}}^{\alpha} D_{0^{+}}^{\alpha} u(t)=u(t)+C_{1} t^{\alpha-1}+C_{2} t^{\alpha-2}+\cdots+C_{n} t^{\alpha-n},
$$

where $C_{i} \in \mathbb{R}, i=1,2, \ldots, n$.

Lemma 4 (see [18]). Suppose that $\mu \in(0,1], v \in(1,2)$ and $u \in C^{2}[0,1], u(0)=u^{\prime}(0)=0$. Then $D_{0^{+}}^{\mu} u \in C[0,1], D_{0^{+}}^{\nu} u \in$ $C[0,1]$, and

$$
\begin{aligned}
& D_{0^{+}}^{\mu} u(t)=\frac{1}{\Gamma(2-\mu)} \int_{0}^{t}(t-s)^{1-\mu} u^{\prime \prime}(s) d s, \\
& D_{0^{+}}^{v} u(t)=\frac{1}{\Gamma(2-\nu)} \int_{0}^{t}(t-s)^{1-v} u^{\prime \prime}(s) d s .
\end{aligned}
$$

Lemma 5. If $\left(H_{1}\right)$ holds, then for any $y \in L^{1}(0,1)$ and $\alpha \epsilon$ $(3,4]$ the boundary value problem

$$
\begin{gathered}
-D_{0^{+}}^{\alpha} x(t)=\phi_{q_{i}}(y(t)), \quad 0<t<1, \\
x(0)=x^{\prime}(0)=x^{\prime \prime}(0)=0, \\
x^{\prime \prime}(1)=(\alpha-1)(\alpha-2) \int_{0}^{1} x(s) d \xi_{i}(s)
\end{gathered}
$$

has a unique solution

$$
x(t)=\int_{0}^{1} H_{i}(t, s) \phi_{q_{i}}(y(s)) d s, \quad i=1,2,
$$

where

$$
\begin{aligned}
& H_{i}(t, s)=G_{\alpha}(t, s)+\frac{t^{\alpha-1}}{1-A_{i}} \int_{0}^{1} G_{\alpha}(\tau, s) d \xi_{i}(\tau), \\
& i=1,2, \\
& G_{\alpha}(t, s) \\
& \quad=\frac{1}{\Gamma(\alpha)} \begin{cases}t^{\alpha-1}(1-s)^{\alpha-3}-(t-s)^{\alpha-1}, & 0 \leq s \leq t \leq 1, \\
t^{\alpha-1}(1-s)^{\alpha-3}, & 0 \leq t \leq s \leq 1 .\end{cases}
\end{aligned}
$$

Proof. By Lemma 3, we can see that

$$
\begin{aligned}
x(t)= & -I_{0^{+}}^{\alpha}\left(\phi_{q_{i}}(y(t))\right)+C_{1} t^{\alpha-1}+C_{2} t^{\alpha-2} \\
& +C_{3} t^{\alpha-3}+C_{4} t^{\alpha-4} .
\end{aligned}
$$

Considering that solutions satisfy $x(0)=x^{\prime}(0)=x^{\prime \prime}(0)=0$ and $x^{\prime \prime}(1)=(\alpha-1)(\alpha-2) \int_{0}^{1} x(s) d \xi_{i}(s)$, we can get $C_{2}=$ $C_{3}=C_{4}=0$ and $C_{1}=\left(\int_{0}^{1}(1-s)^{\alpha-3} \phi_{q_{i}}(y(s)) d s-\int_{0}^{1} \int_{0}^{s}(s-\right.$ $\left.\tau)^{\alpha-1} \phi_{q_{i}}(y(\tau)) d \tau d \xi_{i}(s)\right) / \Gamma(\alpha)\left(1-A_{i}\right)$. As a result,

$$
\begin{aligned}
x(t)= & \frac{t^{\alpha-1}}{\Gamma(\alpha)\left(1-A_{i}\right)}\left[\int_{0}^{1}(1-s)^{\alpha-3} \phi_{q_{i}}(y(s)) d s\right. \\
& -\quad-\frac{1}{\Gamma(\alpha)} \int_{0}^{t}(t-s)^{\alpha-1} \phi_{q_{i}}(y(s)) d s \\
= & \int_{0}^{1} G_{\alpha}(t, s) \phi_{q_{i}}(y(s)) d s \\
& +\frac{t^{\alpha-1}}{1-A_{i}} \iint_{0}^{1} G_{\alpha}(\tau, s) \phi_{q_{i}}(y(s)) d \xi_{i}(\tau) d s \\
= & \int_{0}^{1} H_{i}(t, s) \phi_{q_{i}}(y(s)) d s .
\end{aligned}
$$


Lemma 6 (see [14]). If $\left(H_{1}\right)$ holds, then for any $z \in L^{1}(0,1)$ and $\beta \in(1,2]$ the boundary value problem

$$
\begin{gathered}
-D_{0^{+}}^{\beta} x(t)=z(t), \quad 0<t<1, \\
y(0)=0, \quad x(1)=\int_{0}^{1} y(s) d \eta_{i}(s)
\end{gathered}
$$

has a unique solution

$$
y(t)=\int_{0}^{1} K_{i}(t, s) z(s) d s, \quad i=1,2,
$$

where

$$
\begin{aligned}
& K_{i}(t, s)=G_{\beta}(t, s)+\frac{t^{\beta-1}}{1-B_{i}} \int_{0}^{1} G_{\beta}(\tau, s) d \eta_{i}(\tau), \\
& i=1,2, \\
& G_{\beta}(t, s) \\
& \quad=\frac{1}{\Gamma(\beta)} \begin{cases}t^{\beta-1}(1-s)^{\beta-1}-(t-s)^{\beta-1}, & 0 \leq s \leq t \leq 1, \\
t^{\beta-1}(1-s)^{\beta-1}, & 0 \leq t \leq s \leq 1 .\end{cases}
\end{aligned}
$$

Lemma 7 (see [18]). Let $G_{\alpha}$ and $G_{\beta}$ be as defined in (12) and (18). Then

(1) $G_{\alpha}(t, s) \in C([0,1] \times[0,1])$ and $G_{\alpha}(t, s)>0$ on $(0,1) \times$ $(0,1)$

(2) $e_{\alpha}(1-t) e_{\alpha}(s) / \Gamma(\alpha) \leq G_{\alpha}(t, s) \leq 1 / \Gamma(\alpha)$ for $(t, s) \epsilon$ $[0,1] \times[0,1]$

(3) $(\partial / \partial t) G_{\alpha}(t, s) \in C([0,1] \times[0,1])$ and $(\partial / \partial t) G_{\alpha}(t, s)>$ 0 on $(0,1) \times(0,1)$,

(4) $(\partial / \partial t) G_{\alpha}(t, s) \leq 1 / \Gamma(\alpha-1)$ for $(t, s) \in[0,1] \times[0,1]$,

(5) $\left(\partial^{2} / \partial t^{2}\right) G_{\alpha}(t, s) \in C([0,1] \times[0,1])$ and $\left(\partial^{2} / \partial t^{2}\right)$ $G_{\alpha}(t, s)>0$ on $(0,1) \times(0,1)$,

(6) $\left(\partial^{2} / \partial t^{2}\right) G_{\alpha}(t, s) \leq 1 / \Gamma(\alpha-2)$ for $(t, s) \in[0,1] \times[0,1]$,

(7) $G_{\beta}(t, s) \in C([0,1] \times[0,1])$ and $G_{\beta}(t, s)>0$ on $(0,1) \times$ $(0,1)$

(8) $e_{\beta}(1-t) e_{\beta}(s) / \Gamma(\beta) \leq G_{\beta}(t, s) \leq 1 / \Gamma(\beta)$ for $(t, s) \epsilon$ $[0,1] \times[0,1]$.

Proof. For notational convenience, we denote by

$$
\begin{aligned}
& G_{\alpha}^{*}(t, s) \\
& \quad=\frac{1}{\Gamma(\alpha)} \begin{cases}t^{\alpha-1}(1-s)^{\alpha-1}-(t-s)^{\alpha-1}, & 0 \leq s \leq t \leq 1, \\
t^{\alpha-1}(1-s)^{\alpha-1}, & 0 \leq t \leq s \leq 1 .\end{cases}
\end{aligned}
$$

From paper [19], (7) and (8) hold. Likewise, we have $e_{\alpha}(1-$ t) $e_{\alpha}(s) / \Gamma(\alpha) \leq G_{\alpha}^{*}(t, s)$, since $(1-s)^{\alpha-1} \leq(1-s)^{\alpha-3}$; for $s \in[0,1]$, we have $G_{\alpha}(t, s) \leq G_{\alpha}^{*}(t, s)$, so we have $e_{\alpha}(1-$ t) $e_{\alpha}(s) / \Gamma(\alpha) \leq G_{\alpha}(t, s)$, and from [18], (1)-(6) hold.

Lemma 8. Let $H_{i}, K_{i}$ be as defined in (11) and (17), $i=1,2$. Then
(1) $H_{i}(t, s) \in C([0,1] \times[0,1])$ and $H_{i}(t, s)>0$ on $(0,1) \times$ $(0,1)$,

(2) $e_{\alpha}(1-t) e_{\alpha}(s) / \Gamma(\alpha) \leq H_{i}(t, s) \leq 1 /\left(\Gamma(\alpha)\left(1-a_{i}\right)\right)$ for $(t, s) \in[0,1] \times[0,1]$,

(3) $(\partial / \partial t) H_{i}(t, s) \in C([0,1] \times[0,1])$ and $(\partial / \partial t) H_{i}(t, s)>$ 0 on $(0,1) \times(0,1)$,

(4) $(\partial / \partial t) H_{i}(t, s) \leq 1 /\left(\Gamma(\alpha-1)\left(1-a_{i}\right)\right)$ for $(t, s) \in[0,1] \times$ $[0,1]$

(5) $\left(\partial^{2} / \partial t^{2}\right) H_{i}(t, s) \in C([0,1] \times[0,1])$ and $\left(\partial^{2} / \partial t^{2}\right)$ $H_{i}(t, s)>0$ on $(0,1) \times(0,1)$,

(6) $\left(\partial^{2} / \partial t^{2}\right) H_{i}(t, s) \leq 1 /\left(\Gamma(\alpha-2)\left(1-a_{i}\right)\right)$ for $(t, s) \in[0,1] \times$ $[0,1]$

(7) $K_{i}(t, s) \in C([0,1] \times[0,1])$ and $K_{i}(t, s)>0$ on $(0,1) \times$ $(0,1)$

(8) $e_{\beta}(1-t) e_{\beta}(s) / \Gamma(\beta) \leq K_{i}(t, s) \leq 1 /\left(\Gamma(\beta)\left(1-b_{i}\right)\right)$ for $(t, s) \in[0,1] \times[0,1]$.

Proof. By $\left(\mathrm{H}_{1}\right)$, (11), and Lemma 7, we have $G_{\alpha}(t, s) \leq$ $H_{i}(t, s) \leq(1 / \Gamma(\alpha))\left(1+a_{i} /\left(1-A_{i}\right)\right) \leq 1 /\left(\Gamma(\alpha)\left(1-a_{i}\right)\right)$, so (2) holds; likewise, (1) and (3)-(8) hold.

\section{Auxiliary Regular Problem}

To overcome singularity, we consider the following approximate problem of (1):

$$
\begin{gathered}
D_{0^{+}}^{\beta}\left(\phi_{p_{1}}\left(D_{0^{+}}^{\alpha} u(t)\right)\right) \\
=f_{1 n}\left(t, u(t), v(t), D_{0^{+}}^{\mu} u(t), D_{0^{+}}^{v} v(t)\right), \quad 0<t<1, \\
D_{0^{+}}^{\beta}\left(\phi_{p_{2}}\left(D_{0^{+}}^{\alpha} v(t)\right)\right) \\
=f_{2 n}\left(t, u(t), v(t), D_{0^{+}}^{\mu} u(t), D_{0^{+}}^{v} v(t)\right), \quad 0<t<1, \\
u(0)=u^{\prime}(0)=u^{\prime \prime}(0)=0, \\
u^{\prime \prime}(1)=(\alpha-1)(\alpha-2) \int_{0}^{1} u(s) d \xi_{1}(s), \\
D_{0^{+}}^{\alpha} u(0)=0, \quad \phi_{p_{1}}\left(D_{0^{+}}^{\alpha} u(1)\right)=\int_{0}^{1} \phi_{p_{1}}\left(D_{0^{+}}^{\alpha} u(s)\right) d \eta_{1}(s), \\
v(0)=v^{\prime}(0)=v^{\prime \prime}(0)=0, \\
v^{\prime \prime}(1)=(\alpha-1)(\alpha-2) \int_{0}^{1} v(s) d \xi_{2}(s), \\
D_{0^{+}}^{\alpha} v(0)=0, \quad \phi_{p_{2}}\left(D_{0^{+}}^{\alpha} v(1)\right)=\int_{0}^{1} \phi_{p_{2}}\left(D_{0^{+}}^{\alpha} v(s)\right) d \eta_{2}(s),
\end{gathered}
$$

where $n$ is a positive integer and

$$
\begin{array}{r}
f_{\text {in }}\left(t, x_{1}, x_{2}, x_{3}, x_{4}\right)=f_{i}\left(t, \max \left\{x_{1}, n^{-1}\right\}, \max \left\{x_{2}, n^{-1}\right\},\right. \\
\left.\max \left\{x_{3}, n^{-1}\right\}, \max \left\{x_{4}, n^{-1}\right\}\right) .
\end{array}
$$


Clearly, $f_{\text {in }} \in \operatorname{Car}\left([0,1] \times \mathbb{R}^{+} \times \mathbb{R}^{+} \times \mathbb{R}^{+} \times \mathbb{R}^{+}, \mathbb{R}^{+}\right), i=1,2$. Define a cone $P$ in $E$ as

$$
\begin{gathered}
P=\left\{u \in E: u(0)=u^{\prime}(0)=0, u(t) \geq 0, u^{\prime}(t) \geq 0\right. \\
\left.u^{\prime \prime}(t) \geq 0, t \in[0,1]\right\} .
\end{gathered}
$$

By Lemma 4, we can obtain that

$$
\begin{array}{cc}
D_{0^{+}}^{\mu} u \in C[0,1], & D_{0^{+}}^{v} u \in C[0,1], \\
D_{0^{+}}^{\mu} u(t) \geq 0, & D_{0^{+}}^{v} u(t) \geq 0,
\end{array}
$$

for $u \in P$ and $t \in[0,1]$.

For each $n \in \mathbb{N}^{+}$, let us define operators $T_{1 n}: P \rightarrow E$, $T_{2 n}: P \rightarrow E$ and $T_{n}: P \times P \rightarrow E \times E$ by

$$
\begin{aligned}
& T_{\text {in }}(u, v)(t) \\
& =\int_{0}^{1} H_{i}(t, s) \phi_{q_{i}} \\
& \quad \times\left(\int_{0}^{1} K_{i}(s, \tau) f_{i n}\right. \\
& \left.\quad \times\left(\tau, u(\tau), v(\tau), D_{0^{+}}^{\mu} u(\tau), D_{0^{+}}^{\nu} v(\tau)\right) d \tau\right) d s, \\
& \quad i=1,2,
\end{aligned}
$$

and $T_{n}(u, v)=\left(T_{1 n}(u, v), T_{2 n}(u, v)\right)$. By Lemmas 5 and 6, we know that fixed points of $T_{n}$ are positive solution of the system (20).

Lemma 9. $T_{n}: P \times P \rightarrow P \times P$ is a completely continuous operator.

Proof. We divide the proof into three steps.

Step 1. We prove that $T_{n}: P \times P \rightarrow P \times P$ is well defined.

For all $(u, v) \in P \times P$, let $\rho_{i}(t)=$ $f_{\text {in }}\left(t, u(t), v(t), D_{0^{+}}^{\mu} u(t), D_{0^{+}}^{v} v(t)\right)$. Then by (21) and (23), we have $\rho_{i} \in L^{1}[0,1], i=1,2$. It follows from Lemma 8 that $H_{i},(\partial / \partial t) H_{i},\left(\partial^{2} / \partial t^{2}\right) H_{i}, K_{i}$ are nonnegative and continuous on $[0,1] \times[0,1]$. Therefore, we get $T_{\text {in }}(u, v) \in C^{2}[0,1]$, $T_{\text {in }}(u, v)(0)=T_{\text {in }}^{\prime}(u, v)(0)=0$ and $T_{\text {in }}(u, v)(t) \geq 0$, $T_{\text {in }}^{\prime}(u, v)(t) \geq 0$, and $T_{\text {in }}^{\prime \prime}(u, v)(t) \geq 0$ on $[0,1]$. As a result, $T_{\text {in }}(u, v) \in P$; then we can get $T_{n}(u, v) \in P \times P$.

Step 2. We prove that $T_{n}: P \times P \rightarrow P \times P$ is continuous.

Let $\left\{\left(u_{m}, v_{m}\right)\right\} \subset P \times P$ be a convergent sequence. Suppose that $\lim _{m \rightarrow \infty}\left(u_{m}, v_{m}\right)=(u, v)$. That is, $\lim _{m \rightarrow \infty} u_{m}=u$ and $\lim _{m \rightarrow \infty} v_{m}=v$. Then $\lim _{m \rightarrow \infty} u_{m}^{(j)}(t)=u(t)$ and $\lim _{m \rightarrow \infty} v_{m}^{(j)}(t)=v(t)$ uniformly on $[0,1]$, where $j=0,1,2$. Since, by Lemma 4 , for $\mu \in(0,1]$ and $\nu \in(1,2)$,

$$
\begin{aligned}
& \left|D_{0^{+}}^{\mu} u_{m}(t)-D_{0^{+}}^{\mu} u(t)\right| \\
& \quad \leq \frac{1}{\Gamma(2-\mu)} \int_{0}^{t}(t-s)^{1-\mu}\left|u_{m}^{\prime \prime}(s)-u^{\prime \prime}(s)\right| d s \\
& \quad \leq \frac{\left\|u_{m}^{\prime \prime}-u^{\prime \prime}\right\|_{\infty}}{\Gamma(3-\mu)}, \\
& \left|D_{0^{+}}^{v} v_{m}(t)-D_{0^{+}}^{v} v(t)\right| \\
& \quad \leq \frac{1}{\Gamma(2-v)} \int_{0}^{t}(t-s)^{1-v}\left|v_{m}^{\prime \prime}(s)-v^{\prime \prime}(s)\right| d s \\
& \quad \leq \frac{\left\|v_{m}^{\prime \prime}-v^{\prime \prime}\right\|_{\infty}}{\Gamma(3-v)},
\end{aligned}
$$

we have $\lim _{m \rightarrow \infty} D_{0^{+}}^{\mu} u_{m}(t)=D_{0^{+}}^{\mu} u(t)$ and $\lim _{m \rightarrow \infty} D_{0^{+}}^{v} v_{m}(t)$ $=D_{0^{+}}^{v} v(t)$ uniformly on $[0,1]$. In addition, it follows from (8), for $\mu \in(0,1]$ and $\nu \in(1,2)$, that

$$
\begin{aligned}
& \left\|D_{0^{+}}^{\mu} u_{m}\right\|_{\infty} \\
& \quad \leq \frac{\left\|u_{m}^{\prime \prime}\right\|_{\infty}}{\Gamma(2-\mu)} \int_{0}^{t}(t-s)^{1-\mu} d s \leq \frac{\left\|u_{m}^{\prime \prime}\right\|_{\infty}}{\Gamma(3-\mu)}, \\
& \left\|D_{0^{+}}^{v} v_{m}\right\|_{\infty} \\
& \quad \leq \frac{\left\|v_{m}^{\prime \prime}\right\|_{\infty}}{\Gamma(2-v)} \int_{0}^{t}(t-s)^{1-\nu} d s \leq \frac{\left\|v_{m}^{\prime \prime}\right\|_{\infty}}{\Gamma(3-v)} .
\end{aligned}
$$

Let

$$
\rho_{i m}(t)=f_{i n}\left(t, u_{m}(t), v_{m}(t), D_{0^{+}}^{\mu} u_{m}(t), D_{0^{+}}^{v} v_{m}(t)\right) .
$$

Since $\lim _{m \rightarrow \infty} u_{m}=u, \lim _{m \rightarrow \infty} v_{m}=v$, and $\lim _{m \rightarrow \infty} D_{0^{+}}^{\mu} u_{m}(t)=D_{0^{+}}^{\mu} u(t)$ and $\lim _{m \rightarrow \infty} D_{0^{+}}^{v} v_{m}(t)=$ $D_{0^{+}}^{v} v(t)$ uniformly on $[0,1]$, we have $\lim _{m \rightarrow \infty} \rho_{\text {im }}(t)=\rho_{m}(t)$, for $t \in[0,1]$. Since $\left\{u_{m}\right\}$ and $\left\{v_{m}\right\}$ are bounded in $C^{2}[0,1]$ and $f_{\text {in }} \in \operatorname{Car}\left([0,1] \times \mathbb{R}^{+} \times \mathbb{R}^{+} \times \mathbb{R}^{+} \times \mathbb{R}^{+}, \mathbb{R}^{+}\right)$, inequalities (26) imply that $\left\{D_{0^{+}}^{\mu} u_{m}\right\}$ and $\left\{D_{0^{+}}^{v} v_{m}\right\}$ are bounded in $C[0,1]$. As a result, there exists $\varphi_{i} \in L^{1}[0,1]$ such that $\rho_{i m}(t) \leq \varphi_{i}(t)$, for $t \in[0,1]$ and all $m \in \mathbb{N}^{+}$; from (8) in Lemma 8 , we have $K_{i}(s, \tau) \rho_{i m}(\tau) \leq \varphi_{i}(\tau) / \Gamma(\beta)\left(1-b_{i}\right)$; for $s, \tau \in[0,1]$ and all $m \in \mathbb{N}^{+}$. From the Lebesgue dominated convergence theorem, we can obtain

$$
\lim _{m \rightarrow \infty} \int_{0}^{1} K_{i}(s, \tau) \rho_{i m}(\tau) d \tau=\int_{0}^{1} \begin{gathered}
K_{i}(s, \tau) \rho_{i}(\tau) d \tau, \\
\text { for } s \in[0,1]
\end{gathered}
$$

Since $\phi_{q_{i}}(x)=x^{1 /\left(p_{i}-1\right)}$ is continuous on $[0, \infty)$, we have $\phi_{q_{i}}$ is uniformly continuous on $\left[0,\left\|\varphi_{i}\right\|_{1} /\left(1-b_{i}\right) \Gamma(\beta)\right]$; that is, for all $\epsilon>0, \exists \delta>0$, such that for all $x_{1}, x_{2} \in[0, \infty)$ and $\left|x_{1}-x_{2}\right|<$ $\delta$, we have

$$
\left|\phi_{q_{i}}\left(x_{1}\right)-\phi_{q_{i}}\left(x_{2}\right)\right|<\epsilon .
$$


For (28) we can say that for above $\delta>0$ and $\exists N>0$, for each $m>N$, we have

$$
\left|\int_{0}^{1} K_{i}(s, \tau) \rho_{i m}(\tau) d \tau-\int_{0}^{1} K_{i}(s, \tau) \rho_{i}(\tau) d \tau\right|<\delta .
$$

Since Lemma 8 and $\rho_{i m}(t) \leq \varphi_{i}(t)$, for $t \in[0,1]$, all $m \in \mathbb{N}^{+}$, we have

$$
\begin{aligned}
& 0 \leq \int_{0}^{1} K_{i}(s, \tau) \rho_{i m}(\tau) d \tau \leq \frac{\left\|\varphi_{i}\right\|_{1}}{\left(1-b_{i}\right) \Gamma(\beta)}, \\
& 0 \leq \int_{0}^{1} K_{i}(s, \tau) \rho_{i m}(\tau) d \tau \leq \frac{\left\|\varphi_{i}\right\|_{1}}{\left(1-b_{i}\right) \Gamma(\beta)} .
\end{aligned}
$$

From (29), (30), and (31), we can get

$$
\begin{aligned}
& \left|\phi_{q_{i}}\left(\int_{0}^{1} K_{i}(s, \tau) \rho_{i m}(\tau) d \tau\right)-\phi_{q_{i}}\left(\int_{0}^{1} K_{i}(s, \tau) \rho_{i}(\tau) d \tau\right)\right| \\
& \quad<\epsilon
\end{aligned}
$$

so

$$
\begin{aligned}
& \left|T_{\text {in }}\left(u_{m}, v_{m}\right)(t)-T_{\text {in }}(u, v)(t)\right| \\
& \quad \leq \frac{1}{\Gamma(\alpha)\left(1-a_{i}\right)} \int_{0}^{1} \mid \phi_{q_{i}}\left(\int_{0}^{1} K_{i}(s, \tau) \rho_{i m}(\tau) d \tau\right) \\
& \quad-\phi_{q_{i}}\left(\int_{0}^{1} K_{i}(s, \tau) \rho_{i}(\tau) d \tau\right) \mid d s \\
& <\frac{\epsilon}{\Gamma(\alpha)\left(1-a_{i}\right)},
\end{aligned}
$$

and likewise,

$$
\begin{aligned}
& \left|T_{\text {in }}^{\prime}\left(u_{m}, v_{m}\right)(t)-T_{\text {in }}^{\prime}(u, v)(t)\right|<\frac{\epsilon}{\Gamma(\alpha-1)\left(1-a_{i}\right)}, \\
& \left|T_{\text {in }}^{\prime \prime}\left(u_{m}, v_{m}\right)(t)-T_{\text {in }}^{\prime \prime}(u, v)(t)\right|<\frac{\epsilon}{\Gamma(\alpha-2)\left(1-a_{i}\right)} .
\end{aligned}
$$

Then

$$
\left\|T_{\text {in }}\left(u_{m}, v_{m}\right)-T_{\text {in }}(u, v)\right\|_{*}<\frac{\epsilon}{\Gamma(\alpha-2)\left(1-a_{i}\right)} .
$$

That is, $\lim _{m \rightarrow \infty} T_{i n}\left(u_{m}, v_{m}\right)=T_{i n}(u, v)$, so $\lim _{m \rightarrow \infty} T_{n}$ $\left(u_{m}, v_{m}\right)=T_{n}(u, v)$.

We prove that $T_{n}: P \times P \rightarrow P \times P$ is continuous.

Step 3. We prove that $T_{n}: P \times P \rightarrow P \times P$ is compact; that is, for all $\mathscr{D} \in P \times P$, let $\mathscr{D}$ be a bounded set, and $T_{n}(\mathscr{D})$ is relatively compact in $E \times E$. For notational convenience, we denote by $L_{i}=\left[\left\|\varphi_{i}\right\|_{1} /\left(1-b_{i}\right) \Gamma(\beta)\right]^{1 /\left(p_{i}-1\right)}, i=1,2$.

(I) We prove $T_{n}(\mathscr{D})$ is uniformly bounded in $E \times E$.

For all $\mathscr{D} \in P \times P, \mathscr{D}$ is a bounded set; then $\exists R>0$, such that for all $(u, v) \in \mathscr{D}$, we have $\|(u, v)\| \leq R$; that is, $\|u\|_{*} \leq R$, $\|v\|_{*} \leq R$, and from (26), for $\mu \in(0,1]$ and $\nu \in(1,2)$, we have $\left\|D_{0^{+}}^{\mu} u\right\|_{\infty} \leq R / \Gamma(3-\mu)$ and $\left\|D_{0^{+}}^{v} v\right\|_{\infty} \leq R / \Gamma(3-v)$. Since $f_{\text {in }} \in \operatorname{Car}\left([0,1] \times \mathbb{R}^{+} \times \mathbb{R}^{+} \times \mathbb{R}^{+} \times \mathbb{R}^{+}, \mathbb{R}^{+}\right)$, for some $\varphi_{i} \in$ $L^{1}[0,1]$, such that $f_{\text {in }}\left(t, u(t), v(t), D_{0^{+}}^{\mu} u(t), D_{0^{+}}^{v} v(t)\right) \leq \varphi_{i}(t)$, for $t \in[0,1]$ and all $m \in \mathbb{N}^{+}$, then

$$
\begin{aligned}
& 0 \leq T_{\text {in }}(u, v)(t) \\
& =\int_{0}^{1} H_{i}(t, s) \phi_{q_{i}} \\
& \times\left(\int _ { 0 } ^ { 1 } K _ { i } ( s , \tau ) f _ { \text { in } } \left(\tau, u(\tau), v(\tau), D_{0^{+}}^{\mu} u(\tau),\right.\right. \\
& \left.\left.D_{0^{+}}^{v} v(\tau)\right) d \tau\right) d s \\
& \leq \frac{1}{\Gamma(\alpha)\left(1-a_{i}\right)} \int_{0}^{1} \phi_{q_{i}}\left(\frac{1}{\Gamma(\beta)\left(1-b_{i}\right)} \int_{0}^{1} \varphi_{i}(\tau) d \tau\right) d s \\
& \leq \frac{1}{\Gamma(\alpha)\left(1-a_{i}\right)}\left[\frac{\left\|\varphi_{i}\right\|_{1}}{\left(1-b_{i}\right) \Gamma(\beta)}\right]^{1 /\left(p_{i}-1\right)} \\
& =\frac{L_{i}}{\Gamma(\alpha)\left(1-a_{i}\right)} \text {, } \\
& 0 \leq T_{\text {in }}^{\prime}(u, v)(t) \\
& =\int_{0}^{1} \frac{\partial}{\partial t} H_{i}(t, s) \phi_{q_{i}} \\
& \times\left(\int _ { 0 } ^ { 1 } K _ { i } ( s , \tau ) f _ { \text { in } } \left(\tau, u(\tau), v(\tau), D_{0^{+}}^{\mu} u(\tau),\right.\right. \\
& \left.\left.D_{0^{+}}^{v} v(\tau)\right) d \tau\right) d s \\
& \leq \frac{L_{i}}{\Gamma(\alpha-1)\left(1-a_{i}\right)}, \\
& 0 \leq T_{\text {in }}^{\prime \prime}(u, v)(t) \\
& =\int_{0}^{1} \frac{\partial^{2}}{\partial t^{2}} H_{i}(t, s) \phi_{q_{i}} \\
& \times\left(\int _ { 0 } ^ { 1 } K _ { i } ( s , \tau ) f _ { \text { in } } \left(\tau, u(\tau), v(\tau), D_{0^{+}}^{\mu} u(\tau),\right.\right. \\
& \left.\left.D_{0^{+}}^{v} v(\tau)\right) d \tau\right) d s \\
& \leq \frac{L_{i}}{\Gamma(\alpha-2)\left(1-a_{i}\right)},
\end{aligned}
$$

and then $\left\|T_{\text {in }}(u, v)\right\|_{*} \leq L_{i} / \Gamma(\alpha-2)\left(1-a_{i}\right)$; that is $T_{i n}(\mathscr{D})$ is uniformly bounded in $E \times E$, so $T_{n}(\mathscr{D})$ is also uniformly bounded in $E \times E$.

(II) We prove $T_{n}^{(j)}(\mathscr{D})$ is equicontinuous on $[0,1]$, where $j=1,2$. 
From Lemma 8 , we can get $H_{i}(t, s),(\partial / \partial t) H_{i}(t, s)$, and $\left(\partial^{2} / \partial t^{2}\right) H_{i}(t, s)$ is uniformly continuous on $[0,1]$; that is, for all $\epsilon>0, \exists \delta>0$ such that for all $t_{1}, t_{2}$, $s \in[0,1]$ and $\left|t_{1}-t_{2}\right|<\delta$, we have $\mid H_{i}\left(t_{1}, s\right)-$ $H_{i}\left(t_{2}, s\right)\left|<\epsilon / L_{i},\right|(\partial / \partial t) H_{i}\left(t_{1}, s\right)-(\partial / \partial t) H_{i}\left(t_{2}, s\right) \mid<\epsilon / L_{i}$, and $\left|\left(\partial^{2} / \partial t^{2}\right) H_{i}\left(t_{1}, s\right)-\left(\partial^{2} / \partial t^{2}\right) H_{i}\left(t_{2}, s\right)\right|<\epsilon / L_{i}$.

For all $(u, v) \in \mathscr{D}$, we have

$$
\begin{aligned}
& \left|T_{\text {in }}(u, v)\left(t_{1}\right)-T_{\text {in }}(u, v)\left(t_{2}\right)\right| \\
& \quad \leq \int_{0}^{1}\left|H_{i}\left(t_{1}, s\right)-H_{i}\left(t_{2}, s\right)\right| \phi_{q_{i}} \\
& \quad \times\left(\int_{0}^{1} K_{i}(s, \tau) f_{\text {in }}(\tau, u(\tau), v(\tau),\right. \\
& \left.\left.\quad D_{0^{+}}^{\mu} u(\tau), D_{0^{+}}^{v} v(\tau)\right) d \tau\right) d s \\
& <\frac{\epsilon}{L_{i}} \cdot L_{i}=\epsilon ;
\end{aligned}
$$

likewise

$$
\begin{aligned}
& \left|T_{\text {in }}^{\prime}(u, v)\left(t_{1}\right)-T_{\text {in }}^{\prime}(u, v)\left(t_{2}\right)\right|<\epsilon, \\
& \left|T_{\text {in }}^{\prime \prime}(u, v)\left(t_{1}\right)-T_{\text {in }}^{\prime \prime}(u, v)\left(t_{2}\right)\right|<\epsilon .
\end{aligned}
$$

That is, $T_{i n}^{(j)}(\mathscr{D})$ is equicontinuous on $[0,1]$, so $T_{n}^{(j)}(\mathscr{D})$ is also equicontinuous on $[0,1], j=1,2$.

Applying the Arzelà-Ascoli theorem, $T_{n}(\mathscr{D})$ is relatively compact in $E \times E$, so we prove that $T_{n}: P \times P \rightarrow P \times P$ is compact.

From Steps 1-3, we can get $T_{n}: P \times P \rightarrow P \times P$ is a completely continuous operator.

The proof of Lemma 9 is completed.

Lemma 10 (see $[20,21])$. Let $W$ be a cone in a real Banach space $F, W_{c}=\{x \in W:\|x\|<c\}$, let $\theta$ be a nonnegative continuous concave functional on $W$ such that $\theta(x) \leq\|x\|$ for all $x \in \overline{W_{c}}$, and $W(\theta, b, d)=\{x \in W: \theta(x) \geq b,\|x\| \leq d\}$. Suppose that $A: \overline{W_{c}} \rightarrow \overline{W_{c}}$ is completely continuous and there exist positive constants $0<a<b<d \leq c$ such that

$\left(\mathrm{C}_{1}\right)\{x \in W(\theta, b, d): \theta(x)>b\} \neq \emptyset$ and $\theta(A x)>b$ for $x \in W(\theta, b, d)$,

$\left(\mathrm{C}_{2}\right)\|A x\|<a$ for $x \in \overline{W_{a}}$,

$\left(\mathrm{C}_{3}\right) \theta(A x)>b$ for $x \in W(\theta, b, c)$ with $\|A x\|>d$.

Then $A$ has at least three fixed points $x_{1}, x_{2}$, and $x_{3}$ satisfying

$$
\begin{array}{ll}
\left\|x_{1}\right\|<a, & b<\theta\left(x_{2}\right), \\
\left\|x_{3}\right\|>a, & \theta\left(x_{3}\right)<b .
\end{array}
$$

Remark 11. If $d=c$, then condition $\left(C_{1}\right)$ of Lemma 10 implies condition $\left(C_{3}\right)$ of Lemma 10.
We note $W=P \times P, W_{c}=\{(u, v) \in W:\|(u, v)\|<c\}$, and a nonnegative continuous concave functional $\theta$ on the cone $W$ defined by

$$
\theta(u, v)=\min _{t \in[\zeta, \omega]}|u(t)+v(t)|,
$$

in which $\Gamma(3-\mu) / 2<\zeta<\omega<1$. Since $0<\mu \leq 1$, we get $0<\Gamma(3-\mu) / 2<1$, and $\theta$ is well defined.

For notational convenience, we introduce the following constants:

$$
\begin{array}{r}
h_{\alpha}=\min _{t \in[\zeta, \omega]} e_{\alpha}(1-t), \quad h_{\beta}=\min _{t \in[\zeta, \omega]} e_{\beta}(1-t), \\
m_{i}=\int_{0}^{1} e_{\alpha}(s) \phi_{q_{i}}\left(\int_{\zeta}^{\omega} e_{\beta}(\tau) d \tau\right) d s, \\
n_{i}=\left(\frac{h_{\beta}}{\Gamma(\beta)}\right)^{1 /\left(p_{i}-1\right)}, \\
i=1,2, \\
J_{i}=\Gamma(\alpha-2)\left(1-a_{i}\right)\left[\Gamma(\beta)\left(1-b_{i}\right)\right]^{1 /\left(p_{i}-1\right)}, \\
\Lambda=\left[\left(\frac{h_{\beta}}{\Gamma(\beta)}\right)\left(m_{1} n_{1}+m_{2} n_{2}\right)\right]^{-1}, \\
i=1,2 .
\end{array}
$$
1,2 .

We work with the following conditions on $f_{i}$ in (1), $i=$

$\exists a, b, c$ and $2<a<b<c$, such that $f_{i}$ satisfies the following conditions:

$\left(\mathrm{H}_{2}\right) f_{i}\left(t, x_{1}, x_{2}, x_{3}, x_{4}\right)<\left(J_{i} a\right)^{p_{i}-1}$, for $\left(t, x_{1}, x_{2}, x_{3}, x_{4}\right) \in$ $[0,1] \times(0, a] \times(0, a] \times(0, a / \Gamma(3-\mu)] \times(0, a / \Gamma(3-\nu)]$,

$\left(\mathrm{H}_{3}\right) f_{i}\left(t, x_{1}, x_{2}, x_{3}, x_{4}\right) \leq\left(J_{i} c\right)^{p_{i}-1}$, for $\left(t, x_{1}, x_{2}, x_{3}, x_{4}\right) \in$ $[0,1] \times(0, c] \times(0, c] \times(0, c / \Gamma(3-\mu)] \times(0, c / \Gamma(3-\nu)]$,

$\left(\mathrm{H}_{4}\right) f_{i}\left(t, x_{1}, x_{2}, x_{3}, x_{4}\right)>(\Lambda c)^{p_{i}-1}$, for $\left(t, x_{1}, x_{2}, x_{3}, x_{4}\right) \in$ $[\zeta, \omega] \times[b, c] \times[b, c] \times[(\zeta / \Gamma(3-\mu)) b,(\omega / \Gamma(3-\mu)) c] \times$ $[(\zeta / \Gamma(3-v)) b,(\omega / \Gamma(3-\nu)) c]$,

where $J_{i}, \Lambda$ are defined by (43).

Since $f_{\text {in }}\left(t, x_{1}, x_{2}, x_{3}, x_{4}\right)$ $=$ $f_{i}\left(t, \max \left\{x_{1}, n^{-1}\right\}, \max \left\{x_{2}, n^{-1}\right\}, \max \left\{x_{3}, n^{-1}\right\}, \max \left\{x_{4}, n^{-1}\right\}\right)$, we can get $f_{\text {in }}$ satisfies the following conditions:

$\left(\mathrm{H}_{2}\right)^{\prime} f_{\text {in }}\left(t, x_{1}, x_{2}, x_{3}, x_{4}\right)<\left(J_{i} a\right)^{p_{i}-1}$, for $\left(t, x_{1}, x_{2}, x_{3}, x_{4}\right) \epsilon$ $[0,1] \times[0, a] \times[0, a] \times[0, a / \Gamma(3-\mu)] \times[0, a / \Gamma(3-\nu)]$,

$\left(\mathrm{H}_{3}\right)^{\prime} f_{\text {in }}\left(t, x_{1}, x_{2}, x_{3}, x_{4}\right) \leq\left(J_{i} c\right)^{p_{i}-1}$, for $\left(t, x_{1}, x_{2}, x_{3}, x_{4}\right) \in$ $[0,1] \times[0, c] \times[0, c] \times[0, c / \Gamma(3-\mu)] \times[0, c / \Gamma(3-\nu)]$,

$\left(\mathrm{H}_{4}\right)^{\prime} f_{\text {in }}\left(t, x_{1}, x_{2}, x_{3}, x_{4}\right)>(\Lambda c)^{p_{i}-1}$, for $\left(t, x_{1}, x_{2}, x_{3}, x_{4}\right) \epsilon$ $[\zeta, \omega] \times[b, c] \times[b, c] \times[(\zeta / \Gamma(3-\mu)) b,(\omega / \Gamma(3-\mu)) c] \times$ $[(\zeta / \Gamma(3-v)) b,(\omega / \Gamma(3-\nu)) c]$.

Theorem 12. Assume that $\left(H_{1}\right)-\left(H_{4}\right)$ hold. Then, for $n \in$ $\mathbb{N}^{+}, B V P(20)$ have at least three positive solutions $\left(u_{1 n}, v_{1 n}\right)$, $\left(u_{2 n}, v_{2 n}\right)$, and $\left(u_{3 n}, v_{3 n}\right)$ in $\overline{W_{c}}$, satisfying

$$
\begin{aligned}
& \left\|\left(u_{1 n}, v_{1 n}\right)\right\|<a, \quad b<\min _{t \in[\zeta, \omega]}\left|u_{2 n}(t)+v_{2 n}(t)\right|, \\
& a<\left\|\left(u_{3 n}, v_{3 n}\right)\right\|, \quad \min _{t \in[\zeta, \omega]}\left|u_{3 n}(t)+v_{3 n}(t)\right|<b .
\end{aligned}
$$


Proof. We will show that all conditions of Lemma 10 are satisfied.

Firstly, we prove $T_{n}: \overline{W_{c}} \rightarrow \overline{W_{c}}$ is completely continuous.

For all $(u, v) \in \overline{W_{c}}$, then $\|(u, v)\| \leq c$. So we have $0 \leq$ $u^{(j)}(t) \leq c, 0 \leq v^{(j)}(t) \leq c$, and $t \in[0,1], j=0,1,2$. By (26) we have $0 \leq D_{0^{+}}^{\mu} u(t) \leq c / \Gamma(3-\mu)$ and $0 \leq D_{0^{+}}^{\nu} v(t) \leq c / \Gamma(3-\nu)$. By condition $\left(\mathrm{H}_{3}\right)$, we can get $\left(\mathrm{H}_{3}\right)^{\prime}$ hold, so it follows from condition $\left(\mathrm{H}_{3}\right)$ that

$$
\begin{array}{r}
f_{\text {in }}\left(\tau, u(\tau), v(\tau), D_{0^{+}}^{\mu} u(\tau), D_{0^{+}}^{v} v(\tau)\right) \leq\left(J_{i} c\right)^{p_{i}-1}, \\
\tau \in[0,1]
\end{array}
$$

Thus, for any $(u, v) \in \overline{W_{c}}$, by (45), we have

$$
\begin{aligned}
& \left|T_{\text {in }}(u, v)(t)\right| \\
& =\int_{0}^{1} H_{i}(t, s) \phi_{q_{i}}\left(\int _ { 0 } ^ { 1 } K _ { i } ( s , \tau ) f _ { i n } \left(\tau, u(\tau), v(\tau), D_{0^{+}}^{\mu} u(\tau),\right.\right. \\
& \left.\left.D_{0^{+}}^{v} v(\tau)\right) d \tau\right) d s \\
& \leq \frac{1}{\Gamma(\alpha)\left(1-a_{i}\right)} \\
& \times \int_{0}^{1} \phi_{q_{i}}\left(\int_{0}^{1}\left[\frac{1}{\left(1-b_{i}\right) \Gamma(\beta)}\right]\left(J_{i} c\right)^{p_{i}-1} d \tau\right) d s \\
& =\frac{1}{\Gamma(\alpha)\left(1-a_{i}\right)}\left[\frac{1}{\left(1-b_{i}\right) \Gamma(\beta)}\right]^{1 /\left(p_{i}-1\right)} \cdot J_{i} c \\
& <\frac{J_{i} c}{J_{i}}=c \\
& \left|T_{\text {in }}^{\prime}(u, v)(t)\right| \\
& =\int_{0}^{1} \frac{\partial}{\partial t} H_{i}(t, s) \phi_{q_{i}} \\
& \times\left(\int _ { 0 } ^ { 1 } K _ { i } ( s , \tau ) f _ { \text { in } } \left(\tau, u(\tau), v(\tau), D_{0^{+}}^{\mu} u(\tau),\right.\right. \\
& \left.\left.D_{0^{+}}^{v} v(\tau)\right) d \tau\right) d s \\
& \leq \frac{1}{\Gamma(\alpha-1)\left(1-a_{i}\right)} \\
& \times \int_{0}^{1} \phi_{q_{i}}\left(\int_{0}^{1}\left[\frac{1}{\left(1-b_{i}\right) \Gamma(\beta)}\right]\left(J_{i} c\right)^{p_{i}-1} d \tau\right) d s \\
& =\frac{1}{\Gamma(\alpha-1)\left(1-a_{i}\right)}\left[\frac{1}{\left(1-b_{i}\right) \Gamma(\beta)}\right]^{1 /\left(p_{i}-1\right)} \cdot J_{i} c \\
& <\frac{J_{i} c}{J_{i}}=c \\
& \left|T_{\text {in }}^{\prime \prime}(u, v)(t)\right| \\
& =\int_{0}^{1} \frac{\partial^{2}}{\partial t^{2}} H_{i}(t, s) \phi_{q_{i}} \\
& \times\left(\int _ { 0 } ^ { 1 } K _ { i } ( s , \tau ) f _ { \text { in } } \left(\tau, u(\tau), v(\tau), D_{0^{+}}^{\mu} u(\tau),\right.\right.
\end{aligned}
$$

$$
\begin{gathered}
\left.\left.D_{0^{+}}^{v} v(\tau)\right) d \tau\right) d s \\
\leq \frac{1}{\Gamma(\alpha-2)\left(1-a_{i}\right)} \\
\quad \times \int_{0}^{1} \phi_{q_{i}}\left(\int_{0}^{1}\left[\frac{1}{\left(1-b_{i}\right) \Gamma(\beta)}\right]\left(J_{i} c\right)^{p_{i}-1} d \tau\right) d s \\
=\frac{1}{\Gamma(\alpha-2)\left(1-a_{i}\right)}\left[\frac{1}{\left(1-b_{i}\right) \Gamma(\beta)}\right]^{1 /\left(p_{i}-1\right)} \cdot J_{i} c \\
<\frac{J_{i} c}{J_{i}}=c
\end{gathered}
$$

which means that $\left\|T_{\text {in }}(u, v)\right\|_{*} \leq c$, so we have $\left\|T_{n}(u, v)\right\| \leq c$ and $(u, v) \in \overline{W_{c}}$. Therefore, $T_{n}: \overline{W_{c}} \rightarrow \overline{W_{c}}$. By Lemma 9, we know that $T_{n}: \overline{W_{c}} \rightarrow \overline{W_{c}}$ is completely continuous.

Next, similar to (46), it follows from condition $\left(\mathrm{H}_{2}\right)$ that if $(u, v) \in \overline{W_{a}}$, then $\|T(u, v)\|<a$. So the condition $\left(\mathrm{C}_{2}\right)$ of Lemma 10 holds.

Now, we take $u_{0}(t)=(b+c) / 4$ and $v_{0}(t)=(b+c) / 4$, where $t \in[0,1]$; then

$$
\begin{gathered}
\theta\left(u_{0}, v_{0}\right)=\min _{t \in[\zeta, \omega]}\left|u_{0}(t)+v_{0}(t)\right|=\frac{b+c}{2}>b, \\
\left\|\left(u_{0}, v_{0}\right)\right\|=\frac{b+c}{4}<c,
\end{gathered}
$$

which means that $\left(u_{0}, v_{0}\right) \in W(\theta, b, c)$ and $\theta\left(u_{0}, v_{0}\right)>b$, This proves that $\left\{\left(u_{0}, v_{0}\right) \in W(\theta, b, c): \theta\left(u_{0}, v_{0}\right)>b\right\} \neq \emptyset$.

On the other hand, if $(u, v) \in W(\theta, b, c)$, then $b \leq u^{(j)}(t) \leq$ $c$ and $b \leq v^{(j)}(t) \leq c$, where $t \in[\zeta, \omega]$ and $j=0,1,2$. By Lemma 4 , for $t \in[\zeta, \omega]$, we have

$$
\begin{aligned}
D_{0^{+}}^{\mu} u(t) & =\frac{1}{\Gamma(2-\mu)} \int_{0}^{t}(t-s)^{1-\mu} u^{\prime \prime}(s) d s \\
& \geq \frac{t}{\Gamma(3-\mu)} b \geq \frac{\zeta}{\Gamma(3-\mu)} b, \\
D_{0^{+}}^{\mu} u(t) & =\frac{1}{\Gamma(2-\mu)} \int_{0}^{t}(t-s)^{1-\mu} u^{\prime \prime}(s) d s \\
& \leq \frac{t}{\Gamma(3-\mu)} c \leq \frac{\omega}{\Gamma(3-\mu)} c,
\end{aligned}
$$

and similarly, we can get $(\zeta / \Gamma(3-\nu)) b \leq D_{0^{+}}^{\nu} \nu(t) \leq(\omega / \Gamma(3-$ $\nu)) c$, where $t \in[\zeta, \omega]$. By condition $\left(\mathrm{H}_{4}\right)$, we can get $\left(\mathrm{H}_{4}\right)^{\prime}$ hold, so it follows from condition $\left(\mathrm{H}_{4}\right)$ that

$$
\begin{array}{r}
f_{\text {in }}\left(\tau, u(\tau), v(\tau), D_{0^{+}}^{\mu} u(\tau), D_{0^{+}}^{v} v(\tau)\right)>(\Lambda b)^{p_{i}-1}, \\
\tau \in[\zeta, \omega]
\end{array}
$$


since

$$
\begin{aligned}
\theta\left(T_{n}(u, v)\right) & =\theta\left(T_{1 n}(u, v), T_{2 n}(u, v)\right) \\
& =\min _{t \in[\zeta, \omega]}\left|T_{1 n}(u, v)(t)+T_{2 n}(u, v)(t)\right| \\
& =T_{1 n}(u, v)\left(t_{0}\right)+T_{2 n}(u, v)\left(t_{0}\right),
\end{aligned}
$$

in which $t_{0} \in[\zeta, \omega]$.

Hence, by(49), we have

$$
\begin{aligned}
& \min _{t \in[\zeta, \omega]} T_{\text {in }}(u, v)(t) \\
& =\min _{t \in[\zeta, \omega]} \int_{0}^{1} H_{i}(t, s) \phi_{q_{i}} \\
& \times\left(\int _ { 0 } ^ { 1 } K _ { i } ( s , \tau ) f _ { \text { in } } \left(\tau, u(\tau), v(\tau), D_{0^{+}}^{\mu} u(\tau),\right.\right. \\
& \left.\left.D_{0^{+}}^{v} v(\tau)\right) d \tau\right) d s \\
& \geq \min _{t \in[\zeta, \omega]} \int_{0}^{1} \frac{e_{\alpha}(1-t) e_{\alpha}(s)}{\Gamma(\alpha)} \phi_{q_{i}} \\
& \times\left(\int_{0}^{1} \frac{e_{\beta}(1-s) e_{\beta}(\tau)}{\Gamma(\beta)}\right. \\
& \times f_{\text {in }}\left(\tau, u(\tau), v(\tau), D_{0^{+}}^{\mu} u(\tau),\right. \\
& \left.\left.D_{0^{+}}^{v} v(\tau)\right) d \tau\right) d s \\
& >\min _{t \in[\zeta, \omega]} \frac{e_{\alpha}(1-t)}{\Gamma(\alpha)} \int_{0}^{1} e_{\alpha}(s) \phi_{q_{i}} \\
& \times\left(\int_{\zeta}^{\omega} \frac{h_{\beta}}{\Gamma(\beta)} e_{\beta}(\tau)(\Lambda b)^{p_{i}-1} d \tau\right) d s \\
& =\frac{h_{\alpha}}{\Gamma(\alpha)} \cdot{\frac{h_{\beta}}{\Gamma(\beta)}}^{1 /\left(p_{i}-1\right)} \int_{0}^{1} e_{\alpha}(s) \phi_{q_{i}}\left(\int_{\zeta}^{\omega} e_{\beta}(\tau) d \tau\right) d s \cdot \Lambda b \\
& =\frac{h_{\alpha}}{\Gamma(\alpha)} m_{i} n_{i} \Lambda b
\end{aligned}
$$

so we can get

$$
T_{\text {in }}(u, v)\left(t_{0}\right) \geq \min _{t \in[\zeta, \omega]} T_{\text {in }}(u, v)(t)>\frac{h_{\alpha}}{\Gamma(\alpha)} m_{i} n_{i} \Lambda b .
$$

Hence, by (50), (51), and (52), we have

$$
\begin{aligned}
\theta\left(T_{n}(u, v)\right) & =T_{1 n}(u, v)\left(t_{0}\right)+T_{2 n}(u, v)\left(t_{0}\right) \\
& >\frac{h_{\beta}}{\Gamma(\beta)}\left(m_{1} n_{1}+m_{2} n_{2}\right) \Gamma b=b,
\end{aligned}
$$

which implies that $\theta\left(T_{n}(u, v)\right)$, for $(u, v) \in W(\theta, b, c)$. This shows that condition $\left(\mathrm{C}_{1}\right)$ of Lemma 10 is also satisfied.
By Remark 11, condition $\left(\mathrm{C}_{3}\right)$ of Lemma 10 holds; that is, we show that all conditions of Lemma 10 are satisfied. So we obtain that BVP (20) have at least three positive solutions $\left(u_{1 n}, v_{1 n}\right),\left(u_{2 n}, v_{2 n}\right)$, and $\left(u_{3 n}, v_{3 n}\right)$ in $\overline{W_{c}}$, such that $\left\|\left(u_{1 n}, v_{1 n}\right)\right\|<a, b<\theta\left(u_{2 n}, v_{2 n}\right),\left\|\left(u_{3 n}, v_{3 n}\right)\right\|>a$, and $\theta\left(u_{3 n}, v_{3 n}\right)<b$, hence satisfying

$$
\begin{array}{ll}
\left\|\left(u_{1 n}, v_{1 n}\right)\right\|<a, & b<\min _{t \in[\zeta, \omega]}\left|u_{2 n}(t)+v_{2 n}(t)\right|, \\
a<\left\|\left(u_{3 n}, v_{3 n}\right)\right\|, & \min _{t \in[\zeta, \omega]}\left|u_{3 n}(t)+v_{3 n}(t)\right|<b .
\end{array}
$$

The proof of Theorem 12 is completed.

Lemma 13. Assume that $\left(H_{1}\right)-\left(H_{4}\right)$ hold. Let $\left(u_{n}, v_{n}\right)$ in $\overline{W_{c}}$ be a solution of problem (20). Then the sequence $\left\{\left(u_{n}, v_{n}\right)\right\}$ is relatively compact in $E \times E$.

Proof. Firstly, we prove $\left\{\left(u_{n}, v_{n}\right)\right\}$ is uniformly bounded. Note that

$$
\begin{gathered}
u_{n}(t)=\int_{0}^{1} H_{1}(t, s) \phi_{q_{1}} \\
\quad \times\left(\int _ { 0 } ^ { 1 } K _ { 1 } ( s , \tau ) f _ { 1 n } \left(\tau, u(\tau), v(\tau), D_{0^{+}}^{\mu} u(\tau),\right.\right. \\
\left.\left.D_{0^{+}}^{\nu} v(\tau)\right) d \tau\right) d s, \\
v_{n}(t)=\int_{0}^{1} H_{2}(t, s) \phi_{q_{2}} \\
\times\left(\int _ { 0 } ^ { 1 } K _ { 2 } ( s , \tau ) f _ { 2 n } \left(\tau, u(\tau), v(\tau), D_{0^{+}}^{\mu} u(\tau),\right.\right. \\
\left.\left.D_{0^{+}}^{\nu} v(\tau)\right) d \tau\right) d s .
\end{gathered}
$$

Since $\left(u_{n}, v_{n}\right)$ is in $\overline{W_{c}}$, we can get $\left\{\left(u_{n}, v_{n}\right)\right\}$ is uniformly bounded.

Next, we prove $\left\{\left(u_{n}, v_{n}\right)\right\}$ is equicontinuous on $[0,1]$. Since $\left(u_{n}, v_{n}\right)$ is in $\overline{W_{c}}$, we have $\left\|u_{n}\right\|_{*} \leq c,\left\|v_{n}\right\|_{*} \leq c$, and by (26), we have $0 \leq D_{0^{+}}^{\mu} u(t) \leq c / \Gamma(3-\mu)$ and $0 \leq D_{0^{+}}^{v} v(t) \leq$ $c / \Gamma(3-\mu)$. From Lemma 8 , we can get $H_{1}(t, s),(\partial / \partial t) H_{1}(t, s)$, and $\left(\partial^{2} / \partial t^{2}\right) H_{1}(t, s)$ are uniformly continuous on $[0,1]$, that is, for all $\epsilon>0, \exists \delta>0$ such that for all $t_{1}$ and $t_{2}, s \in[0,1]$, $\left|t_{1}-t_{2}\right|<\delta$, and we have

$$
\begin{gathered}
\left|H_{1}\left(t_{1}, s\right)-H_{1}\left(t_{2}, s\right)\right|<\epsilon, \\
\left|\frac{\partial}{\partial t} H_{1}\left(t_{1}, s\right)-\frac{\partial}{\partial t} H_{1}\left(t_{2}, s\right)\right|<\epsilon, \\
\left|\frac{\partial^{2}}{\partial t^{2}} H_{1}\left(t_{1}, s\right)-\frac{\partial^{2}}{\partial t^{2}} H_{1}\left(t_{2}, s\right)\right|<\epsilon .
\end{gathered}
$$


By $\left(\mathrm{H}_{2}\right)$ and Lemma 8 , we have

$$
\begin{aligned}
& \left|u_{n}\left(t_{1}\right)-u_{n}\left(t_{2}\right)\right| \\
& \leq \int_{0}^{1}\left|H_{1}\left(t_{1}, s\right)-H_{1}\left(t_{2}, s\right)\right| \phi_{q_{1}} \\
& \quad \times\left(\int _ { 0 } ^ { 1 } K _ { 1 } ( s , \tau ) f _ { 1 n } \left(\tau, u(\tau), v(\tau), D_{0^{+}}^{\mu} u(\tau),\right.\right. \\
& \left.\left.\quad D_{0^{+}}^{\nu} v(\tau)\right) d \tau\right) d s \\
& <\epsilon \phi_{q_{1}}\left(\frac{1}{\left(1-b_{1}\right) \Gamma(\beta)}\left(J_{1} a\right)^{p_{1}-1}\right) \\
& =\frac{J_{1} a}{\left[\left(1-b_{1}\right) \Gamma(\beta)\right]^{1 /\left(p_{1}-1\right)}} \epsilon,
\end{aligned}
$$

and similar to (57), we get

$$
\begin{aligned}
& \left|u_{n}^{\prime}\left(t_{1}\right)-u_{n}^{\prime}\left(t_{2}\right)\right|<\frac{J_{1} a}{\left[\left(1-b_{1}\right) \Gamma(\beta)\right]^{1 /\left(p_{1}-1\right)}} \epsilon, \\
& \left|u_{n}^{\prime \prime}\left(t_{1}\right)-u_{n}^{\prime \prime}\left(t_{2}\right)\right|<\frac{J_{1} a}{\left[\left(1-b_{1}\right) \Gamma(\beta)\right]^{1 /\left(p_{1}-1\right)}} \epsilon .
\end{aligned}
$$

That is, $\left\{u_{n}^{(j)}\right\}$ is equicontinuous on $[0,1]$ and $j=1,2$. Likewise, we can get $\left\{v_{n}^{(j)}\right\}$ is equicontinuous on $[0,1]$. That is, $\left\{\left(u_{n}^{(j)}, v_{n}^{(j)}\right)\right\}$ is equicontinuous on $[0,1]$ and $j=1,2$.

Applying the Arzelà-Ascoli theorem, sequence $\left\{\left(u_{n}, \mathrm{v}_{n}\right)\right\}$ is relatively compact in $E \times E$.

The proof of Lemma 13 is completed.

\section{Main Results}

Theorem 14. Assume that $\left(H_{1}\right)-\left(H_{4}\right)$ hold. Then, for $n \in \mathbb{N}^{+}$, $B V P(1)$ have at least three positive solutions $\left(u_{1}, v_{1}\right),\left(u_{2}, v_{2}\right)$, and $\left(u_{3}, v_{3}\right)$ in $\overline{W_{c}}$, satisfying

$$
\begin{aligned}
& \left\|\left(u_{1}, v_{1}\right)\right\| \leq a, \quad b \leq \min _{t \in[\zeta, \omega]}\left|u_{2}(t)+v_{2}(t)\right|, \\
& a \leq\left\|\left(u_{3}, v_{3}\right)\right\|, \quad \min _{t \in[\zeta, \omega]}\left|u_{3}(t)+v_{3}(t)\right| \leq b .
\end{aligned}
$$

Proof. If $\left(\mathrm{H}_{1}\right)-\left(\mathrm{H}_{4}\right)$ hold, by Theorem 12 and Lemma 13, we get BVP $(20)$ have at least three positive solutions $\left(u_{1 n}, v_{1 n}\right)$, $\left(u_{2 n}, v_{2 n}\right)$, and $\left(u_{3 n}, v_{3 n}\right)$ in $\overline{W_{c}}$, satisfying

$$
\begin{aligned}
& \left\|\left(u_{1 n}, v_{1 n}\right)\right\|<a, \quad b<\min _{t \in[\zeta, \omega]}\left|u_{2 n}(t)+v_{2 n}(t)\right|, \\
& a<\left\|\left(u_{3 n}, v_{3 n}\right)\right\|, \quad \min _{t \in[\zeta, \omega]}\left|u_{3 n}(t)+v_{3 n}(t)\right|<b,
\end{aligned}
$$

for $n \in \mathbb{N}^{+}$and for $t \in[0,1]$ and the sequence $\left(u_{1 n}, v_{1 n}\right)$, $\left(u_{2 n}, v_{2 n}\right)$, and $\left(u_{3 n}, v_{3 n}\right)$ are relatively compact in $E \times E$.

We first consider the situation $\left(u_{1 n}, v_{1 n}\right)$; without loss of generality, assume that $\left(u_{1 n}, v_{1 n}\right)$ is convergent in $E \times E$ and $\lim _{n \rightarrow \infty}\left(u_{1 n}, v_{1 n}\right)=\left(u_{1}, v_{1}\right)$; that is, $\lim _{n \rightarrow \infty} u_{1 n}=u_{1}$ and $\lim _{n \rightarrow \infty} v_{1 n}=v_{1}$. Similar to the proof of Lemma 9, we have $\lim _{n \rightarrow \infty} D_{0^{+}}^{\mu} u_{1 n}(t)=D_{0^{+}}^{\mu} u_{1}(t)$ and $\lim _{n \rightarrow \infty} D_{0^{+}}^{v} v_{1 n}(t)=$ $D_{0^{+}}^{\nu} v_{1}(t)$ uniformly on $[0,1]$. Then

$$
\begin{gathered}
\lim _{n \rightarrow \infty} f_{i n}\left(t, u_{1 n}(t), v_{1 n}(t), D_{0^{+}}^{\mu} u_{1 n}(t), D_{0^{+}}^{v} v_{1 n}(t)\right) \\
=f_{i}\left(t, u_{1}(t), v_{1}(t), D_{0^{+}}^{\mu} u_{1}(t), D_{0^{+}}^{v} v_{1}(t)\right) .
\end{gathered}
$$

Since $f_{\text {in }} \in \operatorname{Car}\left([0,1] \times \mathbb{R}^{+} \times \mathbb{R}^{+} \times \mathbb{R}^{+} \times \mathbb{R}^{+}, \mathbb{R}^{+}\right)$, then there exists $\varphi_{i} \in L^{1}[0,1]$, such that

$$
f_{i n}\left(\tau, u_{1 n}(\tau), v_{1 n}(\tau), D_{0^{+}}^{\mu} u_{1 n}(\tau), D_{0^{+}}^{\nu} v_{1 n}(\tau)\right) \leq \varphi_{i}(\tau),
$$

for $\tau \in[0,1]$ and all $m \in \mathbb{N}^{+}$. Hence we can get

$$
\begin{aligned}
& H_{1}(t, s) \phi_{q_{1}} \\
& \quad \times\left(\int_{0}^{1} K_{1}(s, \tau) f_{1 n}\left(\tau, u(\tau), v(\tau), D_{0^{+}}^{\mu} u(\tau), D_{0^{+}}^{v} v(\tau)\right) d \tau\right) \\
& \leq \frac{1}{\Gamma(\alpha)\left(1-a_{1}\right)}\left[\frac{1}{\left(1-b_{1}\right) \Gamma(\beta)}\right]^{1 /\left(p_{1}-1\right)} \phi_{q_{1}}\left(\left\|\varphi_{1}\right\|_{1}\right) .
\end{aligned}
$$

By (61), (63), and the Lebesgue dominated convergence theorem, we can obtain

$$
\begin{gathered}
\lim _{n \rightarrow \infty} \int_{0}^{1} K_{1}(s, \tau) f_{1 n}\left(\tau, u_{1 n}(\tau), v_{1 n}(\tau), D_{0^{+}}^{\mu} u_{1 n}(\tau),\right. \\
\left.D_{0^{+}}^{v} v_{1 n}(\tau)\right) d \tau \\
=\int_{0}^{1} K_{1}(s, \tau) f_{1}\left(t, u_{1}(\tau), v_{1}(\tau), D_{0^{+}}^{\mu} u_{1}(\tau),\right. \\
\left.D_{1}^{v}(t)=\lim _{n \rightarrow \infty} u_{1 n}(t) \quad v_{1}(\tau)\right) d \tau \\
=\lim _{n \rightarrow \infty} \int_{0}^{1} H_{1}(t, s) \phi_{q_{1}} \\
\times\left(\int _ { 0 } ^ { 1 } K _ { 1 } ( s , \tau ) f _ { 1 n } \left(\tau, u(\tau), v(\tau), D_{0^{+}}^{\mu} u(\tau),\right.\right. \\
\left.\left.D_{0^{+}}^{v} v(\tau)\right) d \tau\right) d s
\end{gathered}
$$




$$
\begin{gathered}
=\int_{0}^{1} H_{1}(t, s) \lim _{n \rightarrow \infty} \phi_{q_{1}} \\
\times\left(\int _ { 0 } ^ { 1 } K _ { 1 } ( s , \tau ) f _ { 1 n } \left(\tau, u(\tau), v(\tau), D_{0^{+}}^{\mu} u(\tau),\right.\right. \\
\left.\left.D_{0^{+}}^{\nu} v(\tau)\right) d \tau\right) d s \\
=\int_{0}^{1} H_{1}(t, s) \phi_{q_{1}} \\
\times\left(\int _ { 0 } ^ { 1 } K _ { 1 } ( s , \tau ) f _ { 1 } \left(t, u_{1}(\tau), v_{1}(\tau), D_{0^{+}}^{\mu} u_{1}(\tau),\right.\right. \\
\left.\left.D_{0^{+}}^{\nu} v_{1}(\tau)\right) d \tau\right) d s .
\end{gathered}
$$

Similarly, we can also get

$$
\begin{aligned}
& v_{1 n}(t)=\int_{0}^{1} H_{2}(t, s) \phi_{q_{2}} \\
& \times\left(\int _ { 0 } ^ { 1 } K _ { 2 } ( s , \tau ) f _ { 2 } \left(t, u_{1}(\tau), v_{1}(\tau), D_{0^{+}}^{\mu} u_{1}(\tau),\right.\right. \\
&\left.\left.D_{0^{+}}^{v} v_{1}(\tau)\right) d \tau\right) d s
\end{aligned}
$$

By Lemmas 5 and 6 , we can obtain $\left(u_{1}, v_{1}\right)$ is positive solution of BVP (1).

Similarly, we can also obtain $\left(u_{2}, v_{2}\right)$ and $\left(u_{3}, v_{3}\right)$ are positive solutions of BVP (1).

Since

$$
\begin{aligned}
& \left\|\left(u_{1 n}, v_{1 n}\right)\right\|<a, \quad b<\min _{t \in[\zeta, \omega]}\left|u_{2 n}(t)+v_{2 n}(t)\right|, \\
& a<\left\|\left(u_{3 n}, v_{3 n}\right)\right\|, \quad \min _{t \in[\zeta, \omega]}\left|u_{3 n}(t)+v_{3 n}(t)\right|<b,
\end{aligned}
$$

for $n \in \mathbb{N}^{+}$and for $t \in[0,1]$, now, we pass to the limit as $n \rightarrow \infty$ in (66). Hence, we have

$$
\begin{aligned}
& \left\|\left(u_{1}, v_{1}\right)\right\| \leq a, \quad b \leq \min _{t \in[\zeta, \omega]}\left|u_{2}(t)+v_{2}(t)\right|, \\
& a \leq\left\|\left(u_{3}, v_{3}\right)\right\|, \quad \min _{t \in[\zeta, \omega]}\left|u_{3}(t)+v_{3}(t)\right| \leq b .
\end{aligned}
$$

The proof of Theorem 14 is completed.

\section{Conflict of Interests}

The authors declare that there is no conflict of interests regarding the publication of this paper.

\section{Acknowledgments}

This work is supported by the National Natural Science Foundation of China (11071001 and 11201109) and the Natural Science Foundation of Anhui Province (1208085MA13).

\section{References}

[1] Z. Bai and H. Lü, "Positive solutions for boundary value problem of nonlinear fractional differential equation," Journal of Mathematical Analysis and Applications, vol. 311, no. 2, pp. 495-505, 2005.

[2] Y. Chang and J. J. Nieto, "Some new existence results for fractional differential inclusions with boundary conditions," Mathematical and Computer Modelling, vol. 49, no. 3-4, pp. 605609, 2009.

[3] W. Deng, "Numerical algorithm for the time fractional FokkerPlanck equation," Journal of Computational Physics, vol. 227, no. 2, pp. 1510-1522, 2007.

[4] R. W. Ibrahim and M. Darus, "Subordination and superordination for univalent solutions for fractional differential equations," Journal of Mathematical Analysis and Applications, vol. 345, no. 2, pp. 871-879, 2008.

[5] S. Ladaci, J. J. Loiseau, and A. Charef, "Fractional order adaptive high-gain controllers for a class of linear systems," Communications in Nonlinear Science and Numerical Simulation, vol. 13, no. 4, pp. 707-714, 2008.

[6] S. Z. Rida, H. M. El-Sherbiny, and A. A. M. Arafa, "On the solution of the fractional nonlinear Schrödinger equation," Physics Letters A: General, Atomic and Solid State Physics, vol. 372, no. 5, pp. 553-558, 2008.

[7] A. Yang and W. Ge, "Positive solutions for boundary value problems of N-Dimension nonlinear fractional differential system," Boundary Value Problems, vol. 2008, Article ID 437453, 15 pages, 2008.

[8] B. Ahmad and J. J. Nieto, "Existence of solutions for nonlocal boundary value problems of higher-order nonlinear fractional differential equations," Abstract and Applied Analysis, vol. 2009, Article ID 494720, 9 pages, 2009.

[9] C. F. Li, X. N. Luo, and Y. Zhou, "Existence of positive solutions of the boundary value problem for nonlinear fractional differential equations," Computers and Mathematics with Applications, vol. 59, no. 3, pp. 1363-1375, 2010.

[10] Y. Zhao, S. Sun, Z. Han, and M. Zhang, "Positive solutions for boundary value problems of nonlinear fractional differential equations," Applied Mathematics and Computation, vol. 217, no. 16, pp. 6950-6958, 2011.

[11] S. Stank, "The existence of positive solutions of singular fractional boundary value problems," Computers and Mathematics with Applications, vol. 62, no. 3, pp. 1379-1388, 2011.

[12] Z. Bai and T. Qiu, "Existence of positive solution for singular fractional differential equation," Applied Mathematics and Computation, vol. 215, no. 7, pp. 2761-2767, 2009.

[13] S. Zhang, "Positive solutions to singular boundary value problem for nonlinear fractional differential equation," Computers and Mathematics with Applications, vol. 59, no. 3, pp. 1300-1309, 2010.

[14] J. Q. Jiang, L. S. Liu, and Y. H. Wu, "Positive solutions for nonlinear fractional differential equations with boundary conditions involving Riemann-Stieltjes integrals," Abstract and Applied Analysis, vol. 2012, Article ID 708192, 21 pages, 2012.

[15] T. Chen, W. Liu, and Z. Hu, "A boundary value problem for fractional differential equation with p-Laplacian operator at resonance," Nonlinear Analysis: Theory, Methods and Applications, vol. 75, no. 6, pp. 3210-3217, 2012.

[16] J. Q. Jiang, L. S. Liu, and Y. H. Wu, "Positive solutions for $\mathrm{p}$-Laplacian fourth-order differential system with integral 
boundary conditions," Discrete Dynamics in Nature and Society, vol. 2012, Article ID 293734, 19 pages, 2012.

[17] K. S. Miller and B. Ross, An Introduction to the Fractional Calculus and Fractional Differential Equation, Wiley, New York, NY, USA, 1993.

[18] Z. Bai and W. Sun, "Existence and multiplicity of positive solutions for singular fractional boundary value problems," Computers and Mathematics with Applications, vol. 63, no. 9, pp. 1369-1381, 2012.

[19] Y. Wang, L. Liu, and Y. Wu, "Positive solutions for a class of fractional boundary value problem with changing sign nonlinearity," Nonlinear Analysis: Theory, Methods and Applications, vol. 74, no. 17, pp. 6434-6441, 2011.

[20] R. W. Leggett and L. R. Williams, "Multiple positive oxed points of nonlinear operators on ordered Banach spaces," Indiana University Mathematics, vol. 28, pp. 673-688, 1979.

[21] D. Guo, J. Sun, and Z. Liu, Functional Method for Nonlinear Ordinary Differential Equation, Shandong Science and Technology Press, Jinan, China, 1995. 


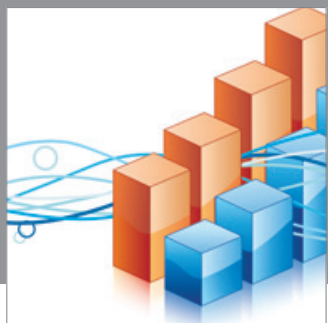

Advances in

Operations Research

mansans

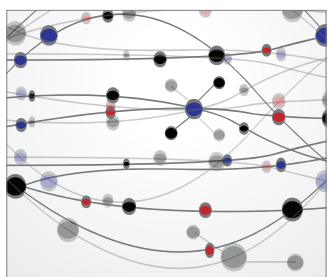

The Scientific World Journal
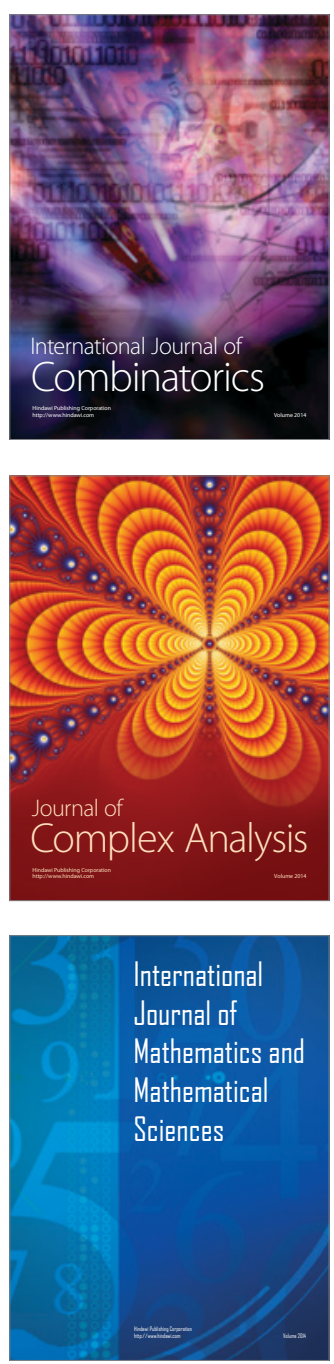
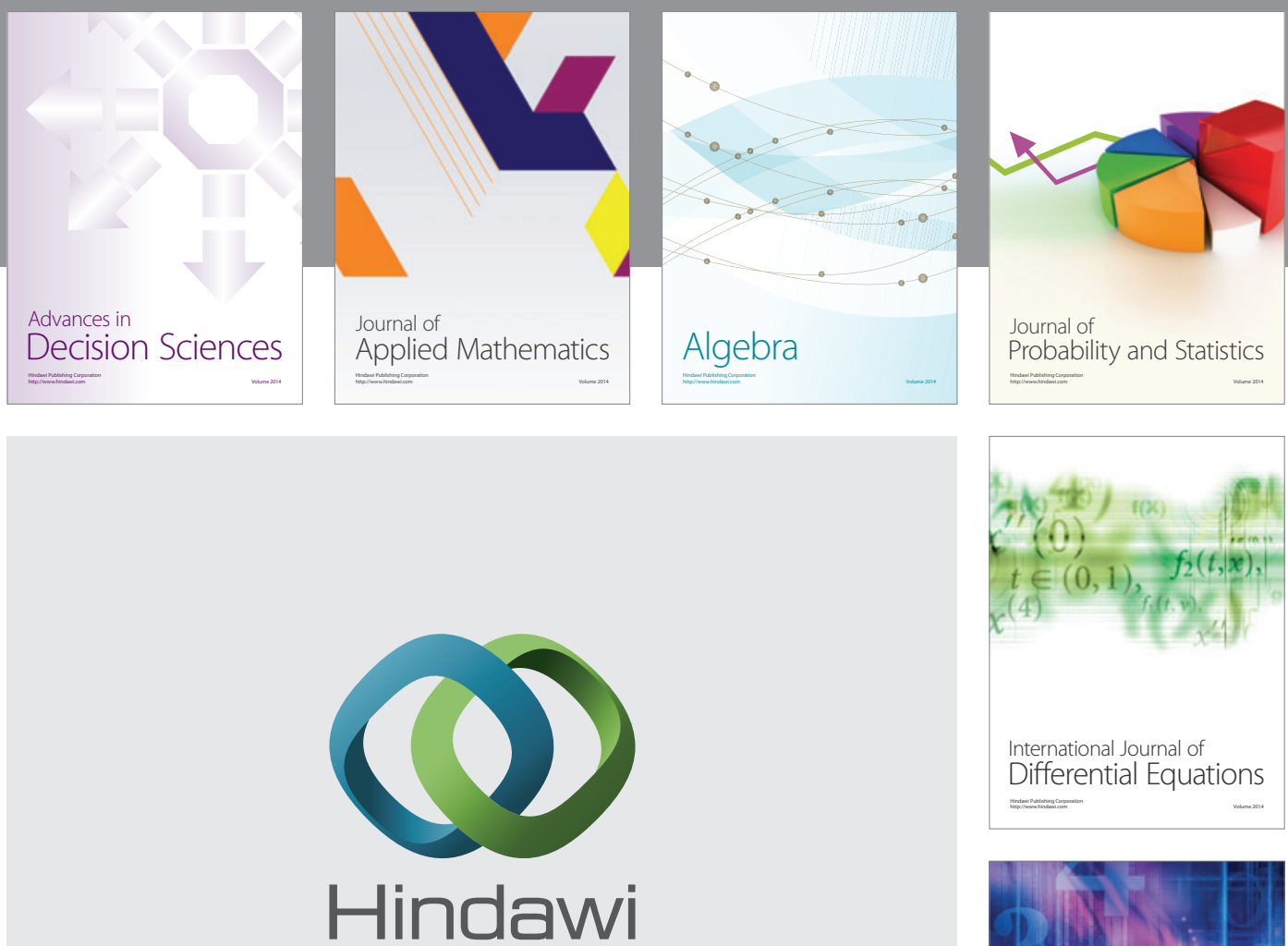

Submit your manuscripts at http://www.hindawi.com
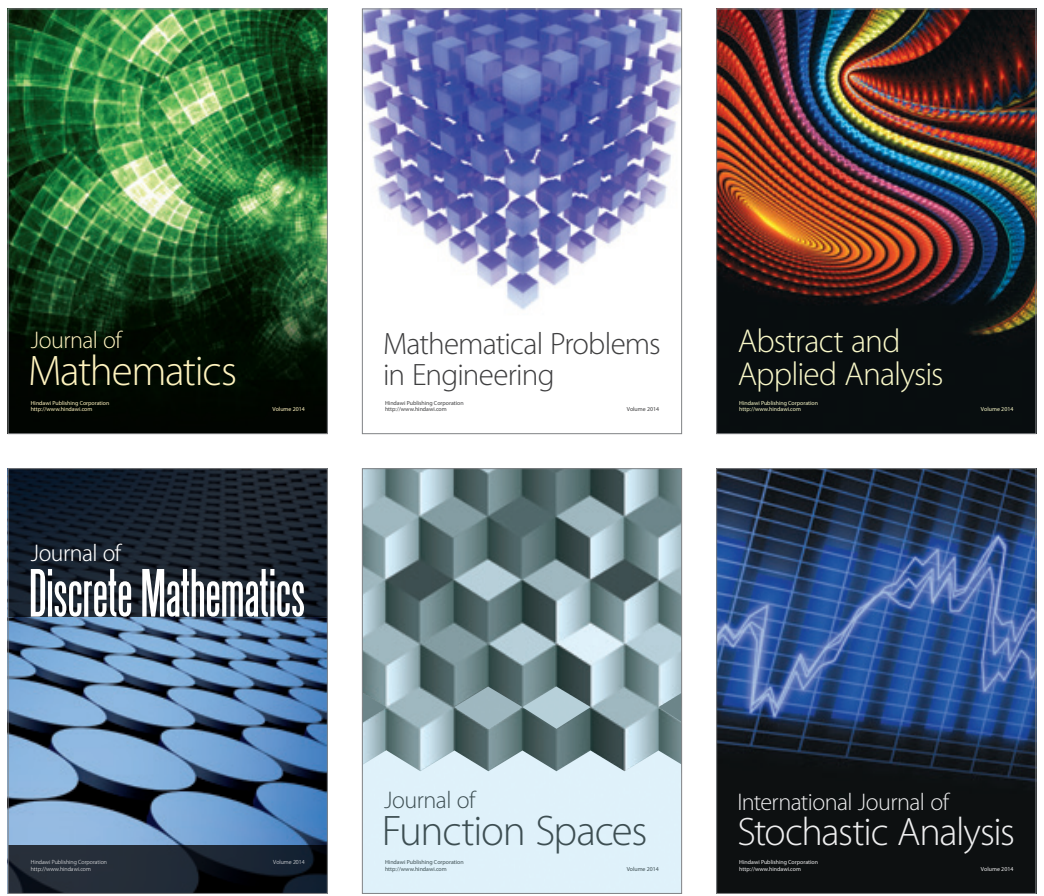

Journal of

Function Spaces

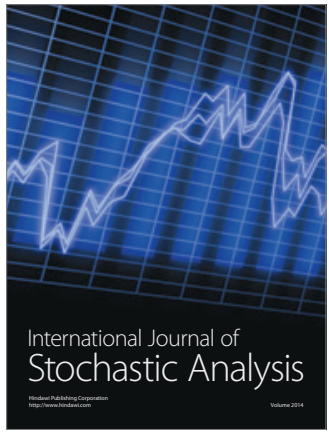

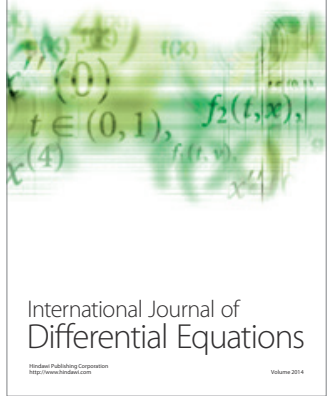
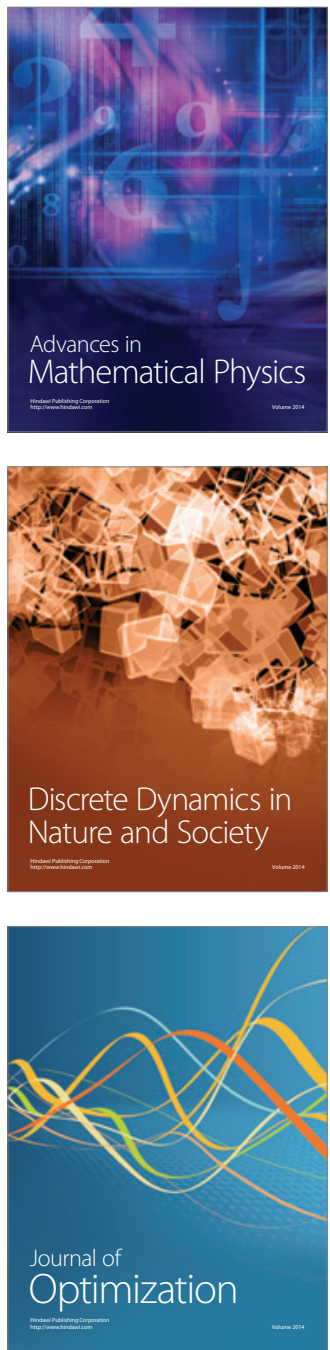